EPJ Web of Conferences 66, 04007 (2014)

DOI: $10.1051 /$ epjconf/ 20146604007

(C) Owned by the authors, published by EDP Sciences, 2014

\title{
Review of Recent Results in Heavy Ion Fluid Dynamics
}

\author{
Laszlo P. Csernai ${ }^{1, a}$ \\ ${ }^{1}$ Dept. of Physics and Technology, University of Bergen, Norway
}

\begin{abstract}
Fluid dynamical phenomena in high energy heavy ion reactions were predicted in the 1970s and still today these are the most dominant and basic observables. With increasing energy and the reach of QGP the low viscosity of the plasma became apparent and this brought a new revolution in the fluid dynamical studies. The high energy and low viscosity made it possible to observe fluctuations up to high multipolarity flow harmonics. This is an obvious, direct proof of the low viscosity of QGP. Many aspects of these fluctuations are under intensive study today. The low viscosity opened ways to observe special fluid dynamical turbulent phenomena. These may arise from random fluctuations, as well as from the global symmetries of peripheral collisions. At LHC energies the angular momentum of the participant matter can reach $10^{6} \hbar$, which leads to rotation and turbulent instabilities, like the Kelvin-Helmholtz instability. Low viscosity ensures that these remain observable at the final freeze-out stages of the collision. Thus new investigations in addition to the standard flow analysis methods became possible. Femtoscopy may also detect rotation and turbulence. Due to the high local thermal vorticity, particle polarization and orbital rotation may reach thermal and mechanical equilibrium. This leads to baryon polarization which, in given directions may be detectable.
\end{abstract}

\section{Introduction}

In high energy heavy ion reactions fluid dynamical phenomena played a fundamental role since the '70-ies and '80-ies [1], and remained the most basic observables until today. Recent theoretical studies indicate that the quark-gluon plasma (QGP) has low viscosity and the minimum value is expected at the phase transition threshold [2]. This low viscosity is directly evidenced by the observation of the azimuthal fluctuation spectrum up to the eights flow harmonics in central collisions [3]. Furthermore, low viscosity can lead to collective instabilities, like the Kelvin Helmholz Instability (KHI), which enhance the observable consequences of particular global flow phenomena.

\section{Random Fluctuations in Fluid Dynamical Processes}

Fluid dynamical (FD) analysis of fluctuations is a great recent success in Relativistic Heavy ion Physics. It is different from the earlier global flow analysis, where the initial symmetries of the collision were exploited, and therefore the final particle distribution there by definition should have been symmetric for reflections across the reaction plane (RP) in peripheral collisions. Therefore the

\footnotetext{
ae-mail: csernai@ift.uib.no
} 
traditional azimuthal angle flow harmonics expansion has only cosine functions with respect to the $\mathrm{RP}$, as the asymmetric sine component should not appear. In addition for symmetric, A+A, colliding systems, the final particle emission have been forward/backward symmetric across the event-by-event (EbE) center of mass (c.m.) point. Of course for such an analysis the RP and c.m. of the exploding system of particles must be identified. As a consequence the rapidity distribution of odd flow harmonics must be odd functions of $y$.

In contrast for random fluctuations in central collisions such restrictions do not apply, the azimuthal dependence can be expanded into odd and even functions, i.e. both sine and cosine. Similarly the rapidity distributions can also be both odd and even functions. This makes the formal expansions somewhat different. In practical evaluations still most of the time the cosine functions are used for the description of azimuthal distributions with an appropriately chosen, multi-polarity $(n)$ dependent azimuth angle, which is then equivalent with using both sine and cosine functions.

The theoretical fits to experimental fluctuation data are also impressive, and adequately chosen initial state fluctuations in the configuration space are able to fit the data with good precision. However, at the same time fluctuations must also arise as a consequence of dynamical fluctuations arising from the FD development, especially if this development is passing close to the critical point [4]. Then the arising critical fluctuations should actually be dominant. This makes the adequacy of the fits based only on initial state shape fluctuations rather questionable.

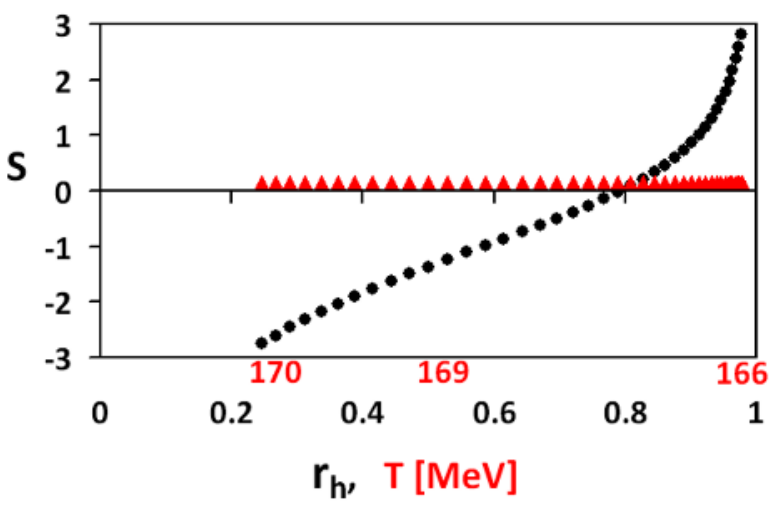

Figure 1. (color online) Skewness as a function of the decreasing temperature (triangles) and the corresponding volume abundance of the hadronic matter (denoted as $r_{h}$, where 1 represents complete hadronization). The temperature, $T$, scale is indicated with the identifiers representing increments of $0.1 \mathrm{MeV}$. Results are for a volume $\Omega=500 \mathrm{fm}^{3}$. From Ref. [6].

The problems of a detailed theoretical analysis is becoming even more complex for peripheral reactions, where both global symmetries and random fluctuations play a strong role. The two types of driving forces behind the dynamics are physically different and due to the nonlinear dynamics the two effects do interfere with each other in a complex way. It would be important to separate the two effects but it by no means is trivial due to the nonlinearity of the development.However due to the different symmetries and asymmetries of the different driving processes, one can achieve at least a reasonable separation of the consequences of random fluctuations (all kinds) from those arising from global dynamics, which are tied to the inherent initial asymmetries in a collision. This is not really 
done in a satisfactory fashion yet. Hence the present day flow analysis of peripheral collisions as if these were only driven by random fluctuations [5], are not complete and cannot be interpreted in a transparent and understandable way.

Finally, the most interesting case for fluctuation studies are the critical fluctuations and critical behaviour at phase transitions, which could provide fundamental information about the properties of the QGP. Fig. 1 shows that even simple phenomenological models predict special fluctuation properties as e.g. the Skewness variation of the energy density during the hadronization of QGP [6]. In order to extract this information reliably, one has to separate and study both physical sources of the fluctuations, and also separate the consequences due to the global flow asymmetries.

Thus the fluctuation studies are at an interesting and promising stage, but a number of open questions require a more thorough and detailed analysis before definite conclusions can be drawn.

\section{Global Collective Flow}

With an increased focus on fluctuations, and the development and use of the methods necessary to study the fluctuations, there is a modest advance in the study of Global Collective flow processes.

Partly the reason is that some of the collective flow signals are becoming smaller with increasing beam energy, like $v_{1}$, and fluctuations dominate the flow. Others, like rotation [7] (see Fig. 2) are becoming stronger. The proper separation of the global collective flow and fluctuations of the flow is not worked out well and not practiced, so these analyses are unconclusive.

On the other hand, other global collective effects are predicted to lead to dominant observable consequences, which are not overshadowed by other processes. They lead to unique observable features, which provide clear evidence for the existence of the global flow pattern.

In this survey talk I would like to draw your attention to two observables: the Differential Hanbury Brown and Twiss (DHBT) method [8] and the measurements of the final baryon polarization in specific directions [9]. The latter are quite sensitive to the angular momentum of the initial state in peripheral heavy ion collisions, and to the specifics of the realization of the large transferred angular momentum in form of local vorticity, shear and other characteristic dynamical properties.

Before doing this I would like to present the global flow characteristics arising from the large transferred angular momentum in peripheral heavy ion reactions. At LHC energies the initial state angular momentum is large, it can reach $10^{6} \hbar c$ in peripheral collisions [10]. This large angular momentum leads to rotation (see Fig. 2) [7] and significant shear velocity and vorticity under favorable conditions, which in turn leads to turbulent instability in the RP (see Fig. 3) [11].

As shown in Fig. 2, the large angular momentum leads to rotation, and to a significant shear and vorticity [12]. The obtained vorticity is more than an order of magnitude higher, $\approx 3 \mathrm{c} / \mathrm{fm}$, than the one obtained from random fluctuations in the transverse plane [13].

We should also consider that the QGP exists for several $\mathrm{fm} / \mathrm{c}$ in high energy heavy ion reactions. Thus, local thermal and fluid dynamical equilibrium can be expected during the reaction, a prerequisite of hydro models. In such equilibrium local vorticity and spin or polarization will equilibrate according to the equipartition theorem [14]. Hence, the measurement of the polarization of emitted particles is a sensitive observable and can help us to diagnose the underlying dynamical state of matter [9].

Turbulent instabilities, like the KHI, may even enhance local rotation significantly, (see Fig. 3), thus polarization may provide a sensitive diagnosis of these phenomena. It is important to observe that the polarization is built up by the flow vorticity, while the random thermal motion decreases it. As a consequence the polarization in a thermally equilibrated system is not proportional to the vorticity of the flow velocity but with the thermal vorticity which is based on the thermal speed constructed 


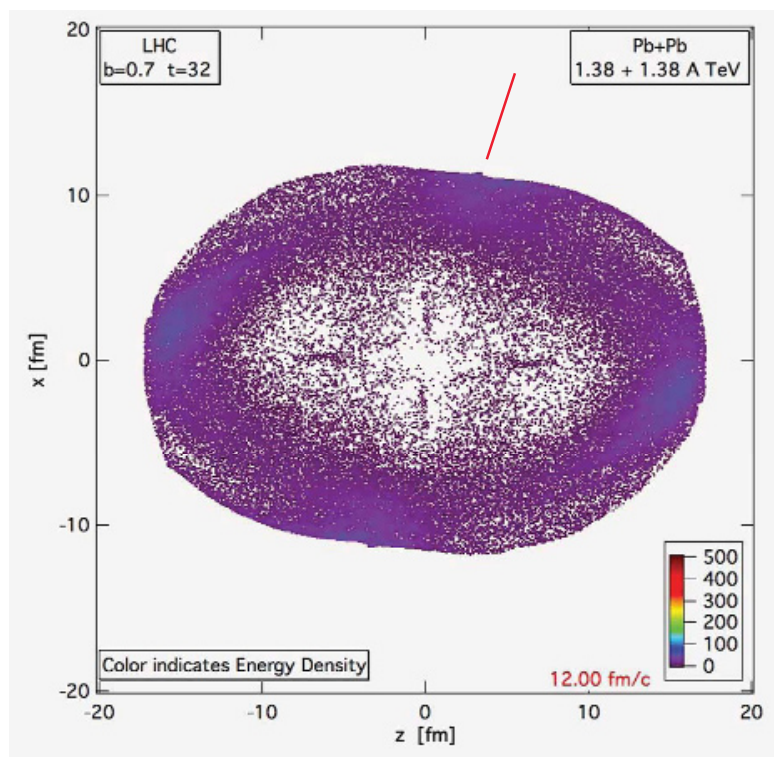

Figure 2. (color online) A moment snapshot of the distribution of the marker particles, i.e. Lagrangian fluid cells (carrying small fixed amount of baryon charge) in the reaction, [x,z] -plane at $12 \mathrm{fm} / \mathrm{c}$ after the start of the fluid dynamical evolution. The color of the points indicates the energy density of the cells. At this time the top energy density of the cells amounts to around $100 \mathrm{GeV} / \mathrm{fm}^{3}$, at four points near the outer surface of the spherically exploding system, which forms a close to spherical shell. This configuration represents approximately the freezeout configuration. The edge point indicated by the red full line on the top indicates the amount of rotation from the start of the fluid dynamical evolution. Based on Ref. [7].

from the flow velocity, $u^{\mu}$ and the temperature, $T$, as:

$$
\beta^{\mu}(x)=\frac{u^{\mu}(x)}{T(x)}
$$

Then absorbing $\hbar$ into $\beta^{\mu}$ we can define thermal vorticity as:

$$
\varpi^{\mu \nu}=\frac{1}{2}\left(\partial^{v} \hat{\beta}^{\mu}-\partial^{\mu} \hat{\beta}^{v}\right)
$$

where $\hat{\beta}^{\mu} \equiv \hbar \beta^{\mu}$. Thereby, $\varpi$ becomes dimensionless. We define an energy density weighted, average vorticity as $\Omega_{z x} \equiv w(z, x) \varpi_{z x}$ so that this weighting does not change the average circulation of the layer, i.e., the sum of the average of the weights over all fluid cells is unity, $\langle w(z, x)\rangle=1$, both if we consider one y-layer only, or if we consider all y-layers. Indexing the cells by $i, k$ in the $x, z$ plane, our weight is proportional to the local energy density $w_{i k} \equiv E_{i k} /\left(E_{t o t} / N_{\text {cell }}\right)$.

The temperature appearing in the denominator of $\beta$ has two effects: (i) the external surface (ii) the beam energy dependence.

The energy averaged thermal vorticity and the consequently arising $\Lambda$ or $\bar{\Lambda}$ polarization [9] are shown in Fig. 4, for a peripheral $\mathrm{Pb}+\mathrm{Pb}$ collision at $\mathrm{LHC}$.

At ultra-relativistic energies the flow velocities do not increase as strongly as the temperature of the system, thus the polarization predicted for RHIC energies is similar or slightly larger than the one 


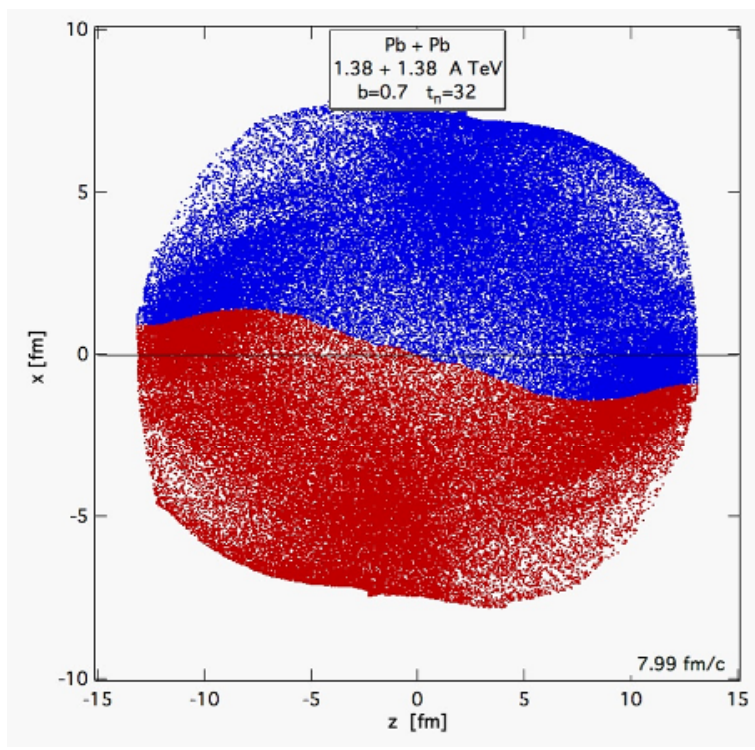

Figure 3. (color online) A snapshot of the spatial distribution of the marker particles in the reaction, $[\mathrm{x}, \mathrm{z}]$, plane at $8 \mathrm{fm} / \mathrm{c}$ after the start of an almost perfect fluid dynamical evolution. At the starting moment the top/bottom part of the exploding system was colored blue/red, respectively. Observe that the boundary has rotated and in addition a non-linear wave has developed, which almost doubles the rotation angle by this time. This large rotation angle leads to observable consequences. This configuration represents approximately the freeze-out configuration. Based on Ref. [11].

presented in Fig. 4 for the LHC. Another observable difference appears at larger energies: due to the larger initial energy density the FO occurs at later times when the vorticity is reduced. Thus, the polarization can be observed equally well at RHIC, as pointed out in Ref. [9]. Hence, the polarization at the highest net baryon densities, i.e. at lower energies, e.g. at FAIR and at NICA [15]. There, the angular momentum is lower. However, the thermal equilibration mechanism is inversely proportional to the temperature, hence, the formation of polarized $\Lambda \mathrm{s}$ or $\bar{\Lambda}$ s should be observed at these baryon densities.

The $\Lambda$ polarization was actually measured by the STAR collaboration in heavy ion reactions at RHIC [16]. However, the polarization was averaged over all azimuthal angles of the emitted $\Lambda \mathrm{s}$ momentum. In Ref. [9] a quantitative prediction of the $\Lambda, \bar{\Lambda}$ polarization is given, for a specific, peripheral centralities and in a given momentum direction.

The polarization in peripheral reaction arises from the rotation in the RP. It is important to identify event by event the RP with its proper orientation, i.e. to identify the projectile and target hemisphere. Fig. 4 indicates, that the maximum polarization occurs into the $x$ direction. The peak is not very sharp in polar angle, however, it is also important to identify the longitudinal c.m. of the system [17], so that the $x$ direction is identified correctly, as shown in Fig. 5.

One should finally mention that the reconstruction of the initial state is essential in these studies. Earlier oversimplified models neglect fundamental processes which lead to shear flow, vorticity and turbulent instabilities. These should be improved to make the initial state models as realistic as possible. 

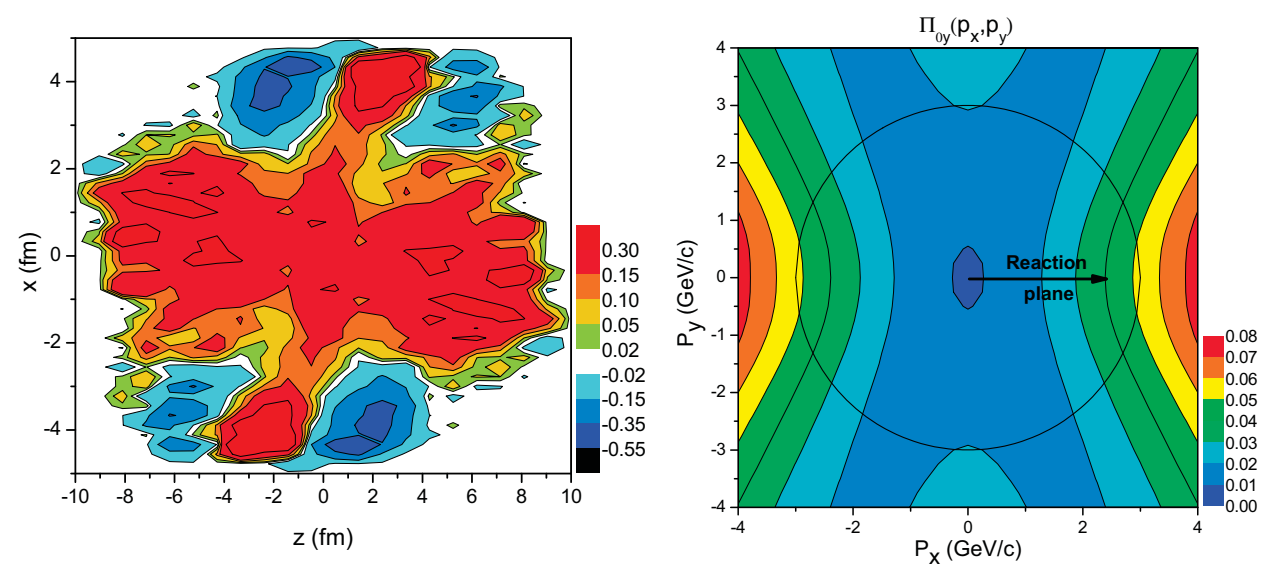

Figure 4. (color online) The weighted average thermal vorticity, $\Omega_{z x}(x, z)$ of the inverse temperature four-vector field $\hat{\beta}^{\mu}$, calculated for all $[x-z]$ layers at $\mathrm{t}=3.56 \mathrm{fm} / \mathrm{c}$, at the freeze-out stage in a $3+1 \mathrm{D}$ fluid dynamical model (Left). The collision energy is $\sqrt{s_{N N}}=2.76 \mathrm{TeV}, b=0.7 b_{\max }$. Note that in this configuration KHI occurs. The cell size is $d x=d y=d z=0.4375 \mathrm{fm}$, while the average weighted vorticity is $\left\langle\Omega_{z x}\right\rangle=0.078$. The arising $\mathrm{y}$-component of the polarization of $\Lambda \mathrm{s}$ emitted in the RP showing peaks in the $\pm x$-direction [9] (Right).

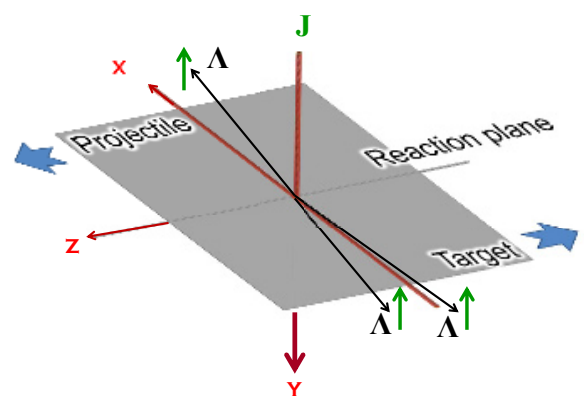

Figure 5. (Color online) Configuration of a peripheral collisions at high energy. The $\Lambda$ polarization points essentially into the direction of the total angular momentum $(-y)$ of the interaction region, orthogonal to the reaction plane. $\Lambda \mathrm{s}$ with the largest polarization are emitted into the $[x, z]$ reaction plane.

\section{Conclusions}

Fluid dynamical models are widely used in the field of heavy ion reactions. For a complete description of the reaction one needs the initial state, and an Equation of State which describes the locally equilibrated matter, and a realistic final freeze out description.

Today we have two main types of fluid dynamical analyses: The first analysing fluctuations, which are best approached in central collisions and thus provides answers to fundamental questions about the phase transition, especially due to critical fluctuations. The other approach is to study global flow effects in peripheral collisions which are more sensitive to the dynamics caused by the pressure gradients, the damping of this dynamics by viscous dissipation, and the level of equilibration itself. The two types of flow processes must clearly be separated from one another, and luckily the difference 
between the symmetry features of central and peripheral collisions provide a possibility to tackle this separation.

The present ultra-relativistic energies open new directions for these studies. In central collisions the multi-polarity of the flow fluctuations opens interesting fundamental studies, while in peripheral collisions new dynamical processes, like rotation and turbulence open new possibilities to study the effects of pressure and viscous damping.

Enlightening discussions with Horst Stöcker are gratefully acknowledged.

\section{References}

[1] L.P. Csernai and J.I. Kapusta, Phys. Rep. 131, 223 (1986); H. Stöcker and W. Greiner, Phys. Rep. 137, 277 (1986); R.B. Clare and D. Strottman, Phys. Rep. 141, 177 (1986); H. Stöcker, J.A. Maruhn, W. Greiner, Phys. Rev. Lett. 44, 725, (1980); L.P. Csernai and H.W. Barz, Z. Phys. A 296 $173(1980)$

[2] L.P. Csernai, J.I. Kapusta, L.D. McLerran, Phys. Rev. Lett. 97, 152303 (2006); and

P.K. Kovtun, D.T. Son, and A.O. Starinets, Phys. Rev. Lett. 94, 111601 (2005)

[3] K. Aamodt et al., (ALICE Collab.) Phys. Rev. Lett. 107, 032301 (2011)

[4] J. I. Kapusta, B. Müller, M. Stephanov Nucl.Phys. A 904-905, 499c-502c (2013)

[5] G. Aad et al. (ATLAS Collaboration), Phys. Rev. C 86, 014907 (2012)

[6] L.P. Csernai, G. Mocanu, Z. Neda, Phys. Rev. C 85, 068201 (2012).

[7] L.P. Csernai, V.K. Magas, H. Stöcker, and D.D. Strottman, Phys. Rev. C 84, 024914 (2011)

[8] L.P. Csernai, S. Velle, D.J. Wang, arXiv:1305.0396; L.P. Csernai, S. Velle, arXiv:1305.0385

[9] F. Becattini, L. Csernai, D. J. Wang, arXiv:1304.4427

[10] V. Vovchenko, D. Anchishkin, L.P. Csernai, Phys. Rev. C 88, 014901 (2013)

[11] L.P. Csernai, D.D. Strottman and Cs. Anderlik, Phys. Rev. C 85, 054901 (2012)

[12] L.P. Csernai, V.K. Magas, D.J. Wang, Phys. Rev. C 87, 034906 (2013)

[13] S. Floerchinger and U.A. Wiedemann, JHEP 11, 100 (2011); and J. Phys. G 38, 124171 (2011)

[14] F. Becattini, V. Chandra, L. Del Zanna, and E. Grossi, arXiv:1303.3431

[15] M. Bleicher, K.A. Bugaev, P. Rau, A.S. Sorin, J. Steinheimer, H. Stoecker, arXiv:1106.3647

[16] B.I. Abelev, et al., (STAR Collaboration), Phys. Rev. C 76, 024915 (2007); Phys. Rev. C 77, 044908 (2008)

[17] L.P. Csernai, G. Eyyubova, and V.K. Magas, Phys. Rev. C 86, 024912 (2012) 
